\title{
ON THE STEINHAUS TILING PROBLEM FOR $\mathbb{Z}^{3}$
}

\author{
DANIEL GOLDSTEIN AND R. DANIEL MAULDIN
}

\begin{abstract}
Steinhaus asked in the 1950's whether there exists a set in $\mathbb{R}^{2}$ meeting every isometric copy of $\mathbb{Z}^{2}$ in precisely one point. Such a "Steinhaus set" was constructed by Jackson and Mauldin. What about $\mathbb{R}^{3}$ ? Is there a subset $S$ of $\mathbb{R}^{3}$ meeting every isometric copy of $\mathbb{Z}^{3}$ in exactly one point? We offer heuristic evidence that the answer is "no".
\end{abstract}

\section{INTRODUCTION}

Steinhaus asked in the late fifties [10, p. 193] if there is a set $S \subseteq \mathbb{R}^{2}$ such that $S$ meets every isometric copy of $\mathbb{Z} \times \mathbb{Z}$ in exactly one point. We call such a set $S$ a Steinhaus set. Equivalently, $S$ is a Steinhaus set if and only if $S$ is a simultaneous transversal for each of the subgroups $R_{\theta} \mathbb{Z}^{2}$, where $R_{\theta}=\left(\begin{array}{c}\cos \theta \sin \theta \\ -\sin \theta \cos \theta\end{array}\right)$. Here a subset $E$ of an abelian group $G$ is a transversal for a subgroup $H<G$ if $E$ meets every coset of $H$ in $G$ in exactly one point. This problem was discussed by Croft in [2] and Croft, Falconer, and Guy in [3, and by Jackson and Mauldin in [7.

Jackson and Mauldin settled this question in [5] and [6].

Theorem 1.1. There is a set $S \subseteq \mathbb{R}^{2}$ that meets every isometric copy $\mathbb{Z} \times \mathbb{Z}$ in exactly one point.

The question of whether the same result holds for $\mathbb{Z}^{3}$ in $\mathbb{R}^{3}$ remains unsolved. It is known (see [8]) that there cannot be a Lebesgue measurable Steinhaus set in $\mathbb{R}^{3}$. It is not known whether there can be a Lebesgue measurable Steinhaus set in $\mathbb{R}^{2}$. However, it is known that no Steinhaus set in $\mathbb{R}^{2}$ is a Borel set or even has the Baire property [7. The analogous questions for $\mathbb{Z}^{n}$ in $\mathbb{R}^{n}$ for $n \geq 4$ have negative answers, as the following observation of the first-named author shows. If $S \subseteq \mathbb{R}^{n}$ were a Steinhaus set for $\mathbb{Z}^{n}(n \geq 4)$, let $x=\left(a_{1}, a_{1}, \ldots, a_{n}\right) \in S \cap \mathbb{Z}^{n}$, and let $y=\left(b_{1}+\right.$ $\left.1 / 2, b_{2}+1 / 2, b_{3}+1 / 2, b_{4}+1 / 2, b_{5}, \ldots, b_{n}\right) \in S \cap\left(\mathbb{Z}^{n}+(1 / 2,1 / 2,1 / 2,1 / 2,0, \ldots, 0)\right)$. Easily $\|x-y\|^{2} \in \mathbb{Z}$. Since every non-negative integer is the sum of four squares, this show that the distance between $x$ and $y$ is the same as a distance between two lattice points in $\mathbb{Z}^{n}$.

We investigate here the three-dimensional version of one of the central results of [6, which involves the idea of extending "partial Steinhaus sets" to larger and larger families of lattices to obtain a Steinhaus set. We give heuristic evidence that this procedure would fail, and that partial Steinhaus sets in $\mathbb{R}^{3}$ don't exist even for rather small families of lattices.

Here is an outline of our heuristic. If a Steinhaus set exists, then, a $p$-partial Steinhaus function (see Definition 3.4) exists for every odd prime $p$. Let $X_{p} \subseteq \mathbb{Z}^{3}$

Date: May 1, 2013.

2000 Mathematics Subject Classification. Primary 28A20; Secondary 52A37, 11H31.

Key words and phrases. lattice points, Steinhaus problem.

The second-named author was supported by National Science Foundation DMS-0700831. 
be the cube of triples $(a, b, c)$ such that $0 \leq a, b, c<p$. By definition, a $p$-partial Steinhaus function is a function $L$ from $X_{p}$ to itself satisfying condition $(+)$ of Lemma 3.3. Given any function $L$ from $X_{p}$ to itself we associate $(p+1) p^{2}$ functions $\pi$ from $G F(p)$ to itself (see Definition 3.6). By Theorem 3.10, $L$ is a $p$-partial Steinhaus function if and only if each of the $(p+1) p^{2}$ associated functions is a permutation.

Here is the numerology. The cube $X_{p}$ has $p^{3}$ points. There are thus $p^{3 p^{3}}$ functions from $X_{p}$ to itself. The probability that a random function from $\{0,1, \ldots, p-1\}$ to itself is a permutation is $p ! / p^{p}$. If the $p^{2}(p+1)$ functions associated to $L$ were random and independently distributed (which they are not), the expected number $N_{p}$ of $p$-partial Steinhaus functions would be

$$
N_{p}=p^{3 p^{3}}\left(\frac{p !}{p^{p}}\right)^{(p+1) p^{2}}
$$

The right-hand side above is less than one for $p=11$ (or for large $p$ by Stirling's approximation). However, if the right-hand side above is less than one, our heuristic argument suggests that no $p$-partial Steinhaus function exists which, if true, would imply that no Steinhaus set exists in dimension 3.

In [5] the following stronger result was shown.

Theorem 1.2. There is a set $S \subseteq \mathbb{R}^{2}$ satisfying:

(1) $S$ meets every isometric copy of $\mathbb{Z} \times \mathbb{Z}$ in exactly one point.

(2) If $x, y \in S$ are distinct then $\|x-y\|^{2} \notin \mathbb{Z}$.

This theorem is stronger since all that was necessary for the Steinhaus problem in dimension 2 was that the squared distance in (2) not be the square of a lattice distance. Our functions $\pi$ arise from extending the use of similar functions in [6 to prove Theorem 1.2.

The authors thank the anonymous referee for comments that led to improvements in the manuscript.

\section{LAtTiCe Distances}

In this paper, a lattice in $\mathbb{R}^{n}$ is an isometric copy of $\mathbb{Z}^{n}$ in $\mathbb{R}^{n}$ By definition, an element of $\left\{\|v\|^{2} \mid v \in \mathbb{Z}^{n}\right\}$ is a squared lattice distance. A squared lattice distance is a sum of $n$ squares of integers.

As already remarked, if $n \geq 4$, then every non-negative integer is a squared lattice distance.

The following useful lemma on squared lattice distances applies in dimension $n=2$ or 3 . Weil [11, p. 292] attributes it to L. Aubry [1. See math overflow 9] for a nice discussion.

Lemma 2.1. Let $n=2$ or 3 . If an integer $N$ is a sum of $n$ rational squares then $N$ is a sum of $n$ integer squares (i.e. is a squared lattice distance).

Proof. We treat the more difficult case $n=3$. The case $n=2$ is treated similarly (and is not used in this paper).

Find a common denominator $m$ for the three rational numbers so that

$$
N=\left(\frac{a}{m}\right)^{2}+\left(\frac{b}{m}\right)^{2}+\left(\frac{c}{m}\right)^{2}
$$


where $a, b, c$ and $m$ are integers. We argue by contradiction. Assume that $N$ is not the sum of three squares of integers. Choose a solution to (2.1) with $m>0$ minimal. By assumption $m>1$.

For the rest of the proof we work in three-dimensional Euclidean space. The point $P=\left(\frac{a}{m}, \frac{b}{m}, \frac{c}{m}\right)$ lies on the sphere $S$ centered at the origin $O$ with squared radius $N$ by (2.1). Let $Z \in \mathbb{Z}^{3}$ be the nearest lattice point to $P$ (choose any if there is more than one). The observation

$$
0<\|P-Z\|^{2} \leq 3 / 4<1 .
$$

has two curious consequences. (1) the line $\overline{P Z}$ is not tangent to $S$ (else $O P Z$ would be a right triangle in which case $\|O-Z\|^{2}=\|O-P\|^{2}+\|P-Z\|^{2}$. This is contradiction since the left hand side is an integer and the right hand side is not.)

It follows from (1) that the line $\overline{P Z}$ meets $S$ in two points, $P$ and say $P^{\prime}$. The second consequence is that $P^{\prime}$ has rational coordinates and yields a solution to (2.1) with denominator $m^{\prime}$ strictly smaller than $m$. Indeed, we can write

$$
P^{\prime}=t P+(1-t) Z
$$

for some real number $t$. Then $\left\|P^{\prime}\right\|^{2}=\|Z+t(P-Z)\|^{2}=\|Z\|^{2}+t Z \cdot(P-Z)+$ $t^{2}\|P-Z\|^{2}$. Since $P^{\prime}$ and $Z$ both lie on $S$ their squared lengths are equal, so that $0=t Z \cdot(P-Z)+t^{2}\|P-Z\|^{2}$, whence

$$
t=-Z \cdot(P-Z) /\|P-Z\|^{2} .
$$

From this we see that the point $m\|Z-P\|^{2} P^{\prime}$ has integer coordinates. But $m\|Z-P\|^{2}$ is a positive integer strictly less than $m$. This contradiction proves the lemma.

\section{Partial Steinhaus sets}

We begin this section with the definition of $m$-partial Steinhaus sets and some elementary remarks. For $m>1$ an integer, set

$$
X_{m}=\{(a, b, c) \mid a, b, c \in \mathbb{Z} \text { and } 0 \leq a, b, c<m\} .
$$

Definition 3.1. A subset $S \subseteq \mathbb{R}^{3}$ is an $m$-partial Steinhaus set if (i) $\left|S \cap\left(\frac{1}{m} x+\mathbb{Z}^{3}\right)\right|=1$ for all $x \in X_{m}$, and (ii) $\|x-z\|^{2}$ is not a squared lattice distance for all distinct $x, z \in S$.

Remark 3.2. (1) Note that by (i), $S$ is of the form $\left\{\frac{1}{m} x+L(x) \mid x \in X_{m}\right\}$ for some function $L: X_{m} \rightarrow \mathbb{Z}^{3}$.

(2) By a straightforward calculation, if $L(x)$ furnishes an m-partial Steinhaus set then so also does $L^{\prime}(x)=L(x)-m \gamma(x)$ for any function $\gamma: X_{m} \rightarrow \mathbb{Z}^{3}$.

(3) If an $m$-partial Steinhaus set exists and $m^{\prime} \mid m$, then an $m^{\prime}$-partial Steinhaus set exists.

We use repeatedly the fact that any vector $v \in \mathbb{Z}^{3}$ is uniquely written:

$$
v=y(v)+m \epsilon(v)
$$

with $y(v) \in X_{m}$ and $\epsilon(v) \in \mathbb{Z}^{3}$. For each coordinate $1 \leq i \leq 3, \epsilon(v)_{i}$ is the quotient and $y(v)_{i}$ the remainder when $v_{i}$ is divided by $m$.

From this fact and Remark $3.2(2)$ we are free to replace $L(x)$ by $y(L(x))$. Thus if an $m$-Steinhaus set exists, we may (and do) assume $L: X_{m} \rightarrow X_{m}$. 
Since $\|L(x)-L(z)\|^{2}$ is a squared lattice distance if it is an integer by Lemma 2.1. we have proved:

Lemma 3.3. We have

(1) For $m>1$ be an integer, let $L: X_{m} \rightarrow \mathbb{Z}^{3}$ be any function. The set $\left\{\frac{1}{m} x+L(x) \mid x \in X_{m}\right\}$ is an m-partial Steinhaus set if and only if

$$
\left\|\left(\frac{1}{m} z+L(z)\right)-\left(\frac{1}{m} x+L(x)\right)\right\|^{2} \quad \text { is not an integer }
$$
for any two distinct elements $x$ and $z$ of $X_{m}$.

(2) If so, then $\left\{\frac{1}{m} x+L(x)+m g(x) \mid x \in X_{m}\right\}$ is an m-partial Steinhaus set for any $g: X_{m} \rightarrow \mathbb{Z}^{3}$. In particular, taking $g(x)=-\epsilon(L(x))$, the set $\left\{\frac{1}{m} x+y(L(x)) \mid x \in X_{m}\right\}$ is an m-partial Steinhaus set.

Definition 3.4. We call a function $L: X_{m} \rightarrow X_{m}$ satisfying $(+)$ an $m$-partial Steinhaus function.

We analyze whether there is such a function $L$ when $m$ is a prime greater than 2 .

Conjecture 3.5. For $p$ a sufficiently large prime, there does not exist a p-partial Steinhaus set in $\mathbb{R}^{3}$.

The truth of Conjecture 3.5 would imply that the Steinhaus problem in $\mathbb{R}^{3}$ has a negative solution. We give heuristic evidence for Conjecture 3.5 in section 4 .

We observe that, whatever $L$ is, if $\left\|\left(\frac{1}{p} z+L(z)\right)-\left(\frac{1}{p} x+L(x)\right)\right\|^{2}$ is an integer, then we must have that $\|z-x\|^{2}$ is divisible by $p$.

We make the following conventions. Let $\Lambda \subseteq X_{p}$ consist of triples $(x, y, z) \neq$ $(0,0,0)$ whose squared norm is divisible by $p$ (and we note that Lemma 3.9 counts the number of such triples). For $\lambda \in \Lambda$, since $\|\lambda\|^{2}$ is divisible by $p$, there is a unique $d=d(\lambda)$ in $\{0, \ldots, p-1\}$ such that $\|\lambda\|^{2} \equiv d p\left(\bmod p^{2}\right)$.

Definition 3.6. Let $L: X_{p} \rightarrow X_{p}$ be any function. Set $F(x)=\frac{1}{p} x+L(x)$. For each $\lambda \in \Lambda$ and $x \in X_{p}$, we define a mapping $\pi_{x}^{\lambda}$ from $\{0, \ldots, p-1\}$ to itself:

$$
\pi_{x}^{\lambda}(t)=\frac{t d(\lambda)}{2}+\lambda \cdot[L(y(x+\lambda t))-\epsilon(x+\lambda t)] \quad(\bmod p) .
$$

For convenience, if $\lambda$ and $x$ are fixed, we shorten the notation to $y_{t}=y(x+\lambda t)$ and $\epsilon_{t}=\epsilon(x+\lambda t)$.

Let $G F(p)$ denote the field of integers modulo $p$. Throughout we identify the elements of $G F(p)$ with the set $\{0,1, \cdots, p-1\}$.

Theorem 3.7. For $p$ an odd prime and $L: X_{p} \rightarrow X_{p}$ any function, the following three statements are equivalent:

(1) For all $x, z \in X_{p}$ with $x \neq z$,

$$
\|F(z)-F(x)\|^{2} \notin \mathbb{Z}
$$

(2) For all $\lambda \in \Lambda$, all $x \in X_{p}$, and all distinct $s, t \in\{0, \cdots p-1\}$,

$$
\left\|F\left(y_{t}\right)-F\left(y_{s}\right)\right\|^{2} \notin \mathbb{Z} \text {. }
$$

(3) For all $\lambda \in \Lambda$ and for all $x \in X_{p}$, the function $\pi_{x}^{\lambda}$ is a permutation of $G F(p)$. 
Note that the relationship between partial Steinhaus sets and permutations was already exploited in [6].

Proof. For the equivalence of (1) and (2) it suffices to note that if $x, z \in X_{p}$ are distinct and $\|F(z)-F(x)\|^{2} \in \mathbb{Z}$ then $z-x=\lambda(\bmod p)$ for some $\lambda \in \Lambda$, thus $z=y_{1}$ and $x=y_{0}$ in the notation defined above.

Fix $\lambda \in \Lambda$ and $x \in X_{p}$. For $0 \leq s, t<p$ we have

$$
\begin{aligned}
& \left\|F\left(y_{t}\right)-F\left(y_{s}\right)\right\|^{2} \notin \mathbb{Z} \\
\Longleftrightarrow & \left\|\left(\frac{y_{t}}{p}+L\left(y_{t}\right)\right)-\left(\frac{y_{s}}{p}-L\left(y_{s}\right)\right)\right\|^{2} \notin \mathbb{Z} \\
\Longleftrightarrow & \|\left(y_{t}-y_{s}\right)+p\left(L\left(y_{t}\right)-L\left(y_{s}\right) \|^{2} \notin p^{2} \mathbb{Z}\right. \\
\Longleftrightarrow & \left\|(t-s) \lambda+p\left[\left(L\left(y_{t}\right)-\epsilon_{t}\right)-\left(L\left(y_{s}\right)-\epsilon_{s}\right)\right]\right\|^{2} \notin p^{2} \mathbb{Z} \\
\Longleftrightarrow & (t-s)^{2} d p+2(t-s) p \lambda \cdot\left[\left(L\left(y_{t}\right)-\epsilon_{t}\right)-\left(L\left(y_{s}\right)-\epsilon_{s}\right)\right] \notin p^{2} \mathbb{Z} \\
\Longleftrightarrow & (t-s)^{2} d+2(t-s) \lambda \cdot\left[\left(L\left(y_{t}\right)-\epsilon_{t}\right)-\left(L\left(y_{s}\right)-\epsilon_{s}\right)\right] \notin p \mathbb{Z} \\
\Longleftrightarrow & 2(t-s)\left(\pi_{x}^{\lambda}(t)-\pi_{x}^{\lambda}(s)\right) \notin p \mathbb{Z} .
\end{aligned}
$$

Since $p$ is odd, if $s \neq t$ then $2(t-s)$ is coprime to $p$, whence

$$
\left\|F\left(y_{t}\right)-F\left(y_{s}\right)\right\|^{2} \notin \mathbb{Z} \Longleftrightarrow \pi_{x}^{\lambda}(t) \not \equiv \pi_{x}^{\lambda}(s) \quad(\bmod p) .
$$

This proves the equivalence of (2) and (3).

If there is a $p$-partial Steinhaus set in dimension 3, then Theorem 3.7 yields a family of permutations of $G F(p)$ denoted $\pi_{x}^{\lambda}$, indexed by $x \in X_{p}$ and $\lambda \in \Lambda$. There are further conditions that this family must satisfy.

Lemma 3.8. The permutations $\pi_{x}^{\lambda}$ satisfy:

(1) For $a \in\{0,1, \cdots, p-1\}$,

$$
\pi_{x}^{\lambda}(t+a)=\frac{a d}{2}+\pi_{x+a \lambda}^{\lambda}(t) \quad(\bmod p) .
$$

(2) For $\alpha \in G F(p)$,

$$
\pi_{x}^{\lambda}(\alpha t)=\pi_{x}^{\alpha \lambda}(t) \quad(\bmod p) .
$$

Proof. Part (1) follows from plugging the identity $x+\lambda(t+a)=(x+a \lambda)+\lambda t$ into (*) and using the definition of $\pi$. Part (2) follows similarly from the identity $x+\lambda(\alpha t)=x+(\alpha \lambda) t$, noting that $\alpha \lambda \in \Lambda$ since $\lambda \in \Lambda$.

The questions arise as to how many permutations $\pi_{x}^{\lambda}$ are needed and what conditions they must satisfy in order that a $p$-partial Steinhaus function $L$ exists. In Theorem 3.10, we show that a particular set of $(p+1) p^{2}$ permutations suffice. Towards showing this, we first note the following.

Let $\mathbb{P}^{2}(G F(p))$ be the projective plane over $G F(p)$.

Lemma 3.9. Let $p$ be a prime, $p>2$. Then

(1) There are exactly $p+1$ triples $(\alpha, \beta, \gamma) \in \mathbb{P}^{2}(G F(p))$ such that $\alpha^{2}+\beta^{2}+\gamma^{2} \equiv$ 0 . 
(2) The set $\Lambda=\left\{(a, b, c) \mid 0 \leq a, b, c<p, a^{2}+b^{2}+c^{2} \equiv 0(\bmod p),(a, b, c) \neq\right.$ $(0,0,0)$ has cardinality $|\Lambda|=p^{2}-1$.

Proof. Let $P=(\alpha, \beta, \gamma) \in \mathbb{P}^{2}(G F(p))$ be a triple satisfying $x^{2}+y^{2}+z^{2} \equiv 0$ $(\bmod p)$. Such a triple exists since the sets $\left\{1+\beta^{2} \mid \beta \in G F(p)\right\}$ and $\left\{-\gamma^{2}\right.$ : $\gamma \in G F(p)\}$, each of cardinality $\frac{p+1}{2}$ have nonempty intersection by the pigeon-hole principle.

Note that the curve $C: x^{2}+y^{2}+z^{2} \equiv 0(\bmod p)$ contains $P$ and has degree 2 . There is one line $\ell_{t}$ through $P$ for each slope $t \in \mathbb{P}^{1}(G F(p))$. Since $C$ has degree 2, each line $\ell_{t}$ meets $C$ in two points: $P$ and say $Q_{t}$ (where $Q_{t}=P$ iff $\ell_{t}$ is tangent to $C$ ). Conversely, for each point $Q$ of $C$ there is a unique line meeting $C$ in $P$ and $Q$. Thus, the set of points of $C$ is in bijection with the set of lines through $P$. There are $p+1$ possible slopes $t$ whence $p+1$ lines whence $p+1$ points on $C$. The line through $P$ with slope $t, 0 \leq t<p$ meets $C$ in the point

$$
\left(\alpha t^{2}-2 \beta t-\alpha,-\beta t^{2}-2 \alpha t+\beta, \gamma\left(1+t^{2}\right)\right) .
$$

The vertical line through $P$ meets $C$ in the point $(\alpha,-\beta, \gamma)$. (Note also that $t=$ $-\alpha / \beta$ is the slope of the tangent line and we get back $P$ again.)

This is precisely the set of $p+1$ triples in $\mathbb{P}^{2}(G F(p))$ that satisfy $x^{2}+y^{2}+z^{2}=$ $0 \in G F(p)$. This proves (i).

For (ii), each projective triple gives rise to $p-1$ triples in $\Lambda$ for a total of $(p-1)(p+1)=p^{2}-1$ by part (i).

To state the next lemma, it is convenient to choose a subset $W \subset \Lambda$ of cardinality $p+1$ representing each projective solution exactly once. By the proof of Lemma 3.9. we have seen that we may take

$$
W=\{(\alpha,-\beta, \gamma)\} \cup\left\{\left(\alpha t^{2}-2 \beta t-\alpha,-\beta t^{2}-2 \alpha t+\beta, \gamma\left(1+t^{2}\right)\right) \mid t \in G F(p)\right\} .
$$

Each $\lambda \in \Lambda$ determines by definition a one-dimensional subspace $\ell_{\lambda}$ of $G F(p)^{3}$. Let $C_{\lambda}$ be a complementary subspace to $\ell_{\lambda}$. Identifying $G F(p)$ with $\{0,1, \cdots, p-1\}$, we view $C_{\lambda}$ as a subset of $X_{p}$. Its cardinality is $p^{2}$.

Theorem 3.10. Let $p>2$ be a prime. Let $L: X_{p} \rightarrow X_{p}$ be any function. Then the following are equivalent:

(1) The set $\left\{\frac{1}{p} x+L(x) \mid x \in X_{p}\right\}$ is a p-partial Steinhaus set.

(2) For all $\lambda \in W$ and $x \in X_{\lambda}$ the map $\pi_{x}^{\lambda}$ is a permutation.

Note that (2) furnishes a collection of $(p+1) p^{2}$ maps from $G F(p)$ to $G F(p)$ that are required to be permutations.

Proof. This follows from Lemma 3.3, Theorem 3.7. Lemma 3.8

\section{Special values of $p$}

In this section we study the number $N_{p}$ of $p$-partial Steinhaus sets, where $p$ is a prime. We show that $N_{3}$ is positive, and give a heuristic argument that $N_{p}$ is 0 for $p \geq 11$. This heuristic argument, if it were in fact valid, would imply a negative solution to the Steinhaus problem in $\mathbb{R}^{3}$.

Recall the definition of a $p$-partial Steinhaus set. Let $L$ be an arbitrary function from a $X_{p}$ to itself. From $L$ is constructed $(p+1) p^{2}$ subsidiary functions $\pi_{x}^{\lambda}$ from $G F(p)$ to $G F(p)$, and $L$ determines a $p$-partial Steinhaus set if and only if all of the subsidiary functions are permutations. 
We show that there is a 3 -partial Steinhaus set (function) in dimension 3 . The function $L$ is determined by its $81=3 \cdot 3^{3}$ coordinate functions in $G F(3)$. In fact, using Theorem 3.10 we find a 3 -partial Steinhaus set where each of the $36=$ $(3+1) 3^{2}$ associated functions from $G F(3)$ to itself is an even permutation (not just a permutation). Note that a sufficient condition for a function $\pi: G F(3) \rightarrow G F(3)$ to be an even permutation is

$$
\pi(1)=\pi(0)+1 \quad \text { and } \quad \pi(2)=\pi(1)+1 .
$$

The condition that a given associated function is even is equivalent to two linear conditions. It is easy (by computer!) to solve this. The 36 associated functions give rise to 72 equations in 81 variables. A computer calculation easily finds the following 3-partial Steinhaus set.

$$
\begin{array}{lll}
(1,2,2) & (2,2,1 / 3) & (1,2,5 / 3) \\
(2,1 / 3,2) & (0,1 / 3,1 / 3) & (2,1 / 3,5 / 3) \\
(1,5 / 3,2) & (2,5 / 3,1 / 3) & (1,5 / 3,5 / 3) \\
(1 / 3,2,2) & (7 / 3,2,1 / 3) & (1 / 3,2,5 / 3) \\
(7 / 3,1 / 3,2) & (4 / 3,1 / 3,1 / 3) & (7 / 3,1 / 3,5 / 3) \\
(1 / 3,5 / 3,2) & (7 / 3,5 / 3,1 / 3) & (1 / 3,5 / 3,5 / 3) \\
(2 / 3,0,0) & (2 / 3,0,1 / 3) & (2 / 3,0,2 / 3) \\
(2 / 3,1 / 3,0) & (2 / 3,1 / 3,1 / 3) & (2 / 3,1 / 3,2 / 3) \\
(2 / 3,2 / 3,0) & (2 / 3,2 / 3,1 / 3) & (2 / 3,2 / 3,2 / 3)
\end{array}
$$

What can one expect for other values of $p$ ? At the time of writing we don't know whether $N_{5}>0$.

We give a heuristic argument. Let $L$ be a function from $X_{p}$ to itself. Associated to any such $L$ are some functions $\pi_{x}^{\lambda}$ from $G F(p)$ to itself. Then $L$ is a $p$-partial Steinhaus function if and only if each of the associated functions from $G F(p)$ to itself is a permutation.

Since $X_{p}$ has cardinality $\left|X_{p}\right|=p^{3}$, there are $\left(p^{3}\right)^{p^{3}}$ functions $L$ from $X_{p}$ to itself. The probability that a random function from $G F(p)$ to itself is a permutation is $p ! / p^{p}$.

If the set of $(p+1) p^{2}$ functions $\pi_{x}^{\lambda}$ was chosen uniformly from the collection of all $(p+1) p^{2}$-tuples of functions from $G F(p)$ to itself, the expected value of $N_{p}$ would be

$$
M_{p}:=p^{3 p^{3}}\left(\frac{p !}{p^{p}}\right)^{(p+1) p^{2}}
$$

Here is a table of $M_{p}$ for some small values of $p$.

\begin{tabular}{r|l}
$p$ & $M_{p}$ \\
\hline 3 & $1.4 E 15$ \\
5 & $5.8 E 49$ \\
7 & 100 \\
11 & $1.1 E-1438$ \\
13 & $4.0 E-3748$
\end{tabular}

By Stirling's approximation, $\log \left(M_{p}\right)=-p^{4}+3.5 p^{3} \log (p)+$ lower order terms. This heuristic reasoning offers some evidence that $N_{p}=0$ for large $p$ (even $p \geq 11$ ). Thus, there would be no $p$-partial Steinhaus function, and the Steinhaus problem in three dimensions would have a negative solution. See [4] for some further work on 
the Steinhaus problem in two dimensions by the NSF-RTG group at the University of North Texas.

\section{REFERENCES}

[1] L. Aubry, Sphinxe-Oedipe 7 (1912), 81-84.

[2] H. T. Croft, Three lattice point problems of Steinhaus, Quart. J. Math. Oxford 33 (1982), 71-83.

[3] H. T. Croft, K. J. Falconer, and R. K. Guy, Unsolved Problems in Geometry, Springer-Verlag, New York, 1991.

[4] D. Harris, S. Jackson, J. Lobe and C. Wahlert, Restricted Steinhaus sets in the plane, preprint.

[5] S. Jackson and R. D. Mauldin, Sets meeting isometric copies of a lattice in exactly one point, Proc. Natl. Acad. Sci, USA, 99 (2002), 15883-15887.

[6] S. Jackson and R. D. Mauldin, On a lattice problem of H. Steinhaus, J. Amer. Math. Soc., 15 (2002), no. 4, 817-856.

[7] S. Jackson and R. D. Mauldin, Survey of the Steinhaus tiling problem, Bull. Symbolic Logic, 9 (2003), 335-361.

[8] M. N. Kolountzakis and T. Wolff, On the Steinhaus tiling problem, Mathematika, 46 (1999), 253-280.

[9] http://mathoverflow.net/questions/3269/intuition-for-the-last-step-in-serres-proof-of-the-three-squares-theorem

[10] W. Sierpiński, Sur un problème de H. Steinhaus concernant les ensembles de points sur le plan, Fund. Math. 46 (1958), 191-194.

[11] Weil, André, Number theory. An approach through history from Hammurapi to Legendre. Reprint of the 1984 edition. Birkhäuser Boston, Inc., Boston, MA, 2007.

Center for Communications Research, 4320 Westerra Court, San Diego, CA 92121

E-mail address: danielgolds@gmail.com

E-mail address: mauldin@unt.edu 